\title{
Interférences
}

Ars scribendi

$6 \mid 2012$

La question de la littérarité

\section{Horacio y la lírica popular}

Jesús Luque Moreno

\section{(2) OpenEdition}

Journals

Edición electrónica

URL: http://journals.openedition.org/interferences/146

DOI: 10.4000/interferences. 146

ISSN: $1777-5485$

Editor

HiSoMA - Histoire et sources des Mondes antiques

Edición impresa

Fecha de publicación: 1 enero 2012

ISSN: 1777-5485

\section{Referencia electrónica}

Jesús Luque Moreno, « Horacio y la lírica popular », Interférences [En ligne], 6 | 2012, mis en ligne le 16 janvier 2018, consulté le 15 septembre 2020. URL : http://journals.openedition.org/interferences/146 ; DOI : https://doi.org/10.4000/interferences. 146

Este documento fue generado automáticamente el 15 septiembre 2020

Tous droits réservés 


\title{
Horacio y la lírica popular
}

\author{
Jesús Luque Moreno
}

1 0. Horacio, como es bien sabido, rechazaba decididamente al vulgo ignorante, manteniéndolo a raya:

Odi profanum vulgus et arceo: carm. III 1,1;

el derecho a tal desprecio, decía, lo tenía concedido junto con el don de la poesía sabia, de la lírica culta:

mihi parva rura et $\|$ spiritum Graiae tenuem Camenae $\|$ Parca non mendax dedit et malignum || spernere volgus: carm. II 16,37;

merecedor de la inmortalidad, él despreciaba el suelo encharcado y los corrillos del vulgo ${ }^{1}$, abriéndose camino hacia el cielo:

virtus recludens inmeritis mori $\|$ caelum negata temptat iter via $\|$ coetusque volgaris et udam || spernit humum fugiente pinna: carm. III 2,21;

alzaba el vuelo y, superando a Ícaro, con su docta frente coronada de hiedra, llegaba a mezclarse con los dioses y a tocar con su cabeza las estrellas:

et album mutor in alitem || superne nascunturque leves $\|$ per digitos umerosque plumae. \|

iam Daedaleo notior Icaro...: carm. II 20,10;

me doctarum hederae praemia frontium $\|$ dis miscent superis ... quodsi me lyricis vatibus

inseres, || sublimi feriam sidera vertice: carm. I 1,29.

2 1. Pues bien, contra lo que cabría esperar, este mismo Horacio, lírico de tan altos vuelos, se mostró buen conocedor de la poesía «vulgar», de los cantos populares; incluso, llegado el caso, no tuvo empacho en presentarse a sí mismo sumergido en la masa de ciudadanos que cantan a coro en una procesión triunfal:

canam recepto || Caesare felix. || teque, dum procedis, io Triumphe, || non semel dicemus, io

Triumphe, II civitas omnis: carm. IV 2,47.

3 1.1. Horacio, por ejemplo, conocía ${ }^{2}$ a Sarmiento (Sarmentus), el liberto ${ }^{3}$ de Mecenas contra el que se cantaron los célebres versus quadrati que han conservado los escolios a Juvenal (schol. Iuv. 5,3):

aliud scriptum habet Sarmentus, aliud populus voluerat.

digna dignis: sic Sarmentus habeat crassas compedes.

rustici, ne nihil agatis: aliquis Sarmentus alliget ${ }^{4}$.

1.2. Horacio citó cantos infantiles en dos ocasiones: en el libro primero de las Epístolas: 
- $\quad$ at pueri ludentes 'rex eris' aiunt, || 'si recte facies': epist. I 1,59 s.

Con los oportunos cambios sintácticos y rítmico-métricos haría aquí referencia el poeta a una canción cuya forma y texto originario, de acuerdo con lo que, comentando el pasaje horaciano, escribió Porfirión ${ }^{5}$, podrían ser los de este septenario trocaico:

rex erit qui recte faciet; qui no faciet, no erit ${ }^{6}$.

De una canción infantil proviene asimismo, según reconocieron ya los comentaristas antiguos ${ }^{7}$, esta otra expresión formular recogida en el Ars poetica:

occupet extremum scabies: ars 417 ,

tras la que se podría reconstruir con Lucien Müller un septenario así:

habeat scabiem quisquis ad me venerit novissimus.

7 2. Y no queda aquí la presencia de la lírica popular en los escritos de Horacio. Ecos de estos cantos populares, «vulgares», pueden oírse no ya en los hexámetros conversacionales de sus cartas, sino nada menos que entre los cuidados versos cultos de sus odas: entre ellos, veladas en mayor o menor grado y entretejidas, como las anteriores, en un entramado rítmico-metrico que les es ajeno ${ }^{9}$, se pueden adivinar citas de versos populares, de versus quadrati (septenarios trocaicos), sobre todo, e incluso en aguna ocasión de fórmulas próximas al viejo saturnio, al horridus saturnius ${ }^{10}$.

2.1. La más conocida y comentada de dichas posibles citas es, sin duda, la recogida en el carmen segundo del libro cuarto:

[33] concines maiore poeta plectro || Caesarem, quandoque trahet ferocis || per sacrum clivum merita decorus $\|$ fronde Sygambros, ||| ... [41] concines laetosque dies et urbis $\|$ publicum ludum super inpetrato \| fortis Augusti reditu forumque \|| litibus orbum. ||| [45] tum meae, si quid loquar audiendum, || vocis accedet bona pars et 'o sol || pulcer, o laudande' canam recepto || Caesare felix. ||| teque, dum procedis, 'io Triumphe', || [50] non semel dicemus, 'io Triumphe', || civitas omnis dabimusque divis || tura benignis;

unos versos que traen a la memoria lo que sobre el propio Augusto escribiría más tarde Suetonio:

Revertentem ex provincia non solum faustis ominibus sed et modulatis carminibus persequebantur ${ }^{11}$.

El contexto, qué duda cabe, sobre todo a partir del verso 45 es el de una procesión triunfal: véase sin más la expresión dum procedis o el grito ritual io Triumphe ${ }^{12}$ :

teque, dum procedis, io Triumphe $\|$ no semel dicemus, io Triumphe;

un colon yambo-trocaico ([i-o-Tri-um-phe]: × - - ), aquí sabiamente incrustrado en el esquema del endecasílabo sáfico; colon que ya antes había hecho presente Horacio en el epodo noveno, al que enseguida prestaré atención.

Se trata con toda probabilidad de un canto coral: concines $^{13}$; el canto de toda la comunidad (dicemus ... civitas omnis dabimusque...) al que Horacio suma su propia voz (meae ... vocis accedet bona pars); no ya su voz de vate consagrado sino la de un ciudadano más que se suma al canto del pueblo. Popularizante es, en efecto, la repetición del io Triumphe ocupando el mismo lugar en versos sucesivos ${ }^{14}$, recurso del que se sirve Horacio tanto en el mencionado epodo como en esta oda. No es, en consecuencia, a uno de sus operosa carmina a lo que aquí se refiere el poeta sino a los versos de un canto popular, expresión más o menos espontánea de la alegría del pueblo, tal como se manifestaba en las celebraciones de los soldados ya desde el triunfo de Camilo ${ }^{15}$.

$11 \mathrm{Y}$, en concreto, lo que dice Horacio que va a cantar en tal ocasión sumergido en la comunidad ciudadana es ' $O$ sol pulcer, o laudande', un dímetro trocaico, un posible 
primer hemistiquio de un posible septenario, el versus quadratus, habitual en tales ocasiones:

$$
\text { --- 1--- }(+-\quad-x-\quad \sim \|) \text {, }
$$

un colon trocaico que aquí ha sido transplantado a la estrofa sáfica y sabiamente entretejido en el esquema del endecasílabo ${ }^{16}$ :

$$
\text { - } \| \text { - _. _ / - - - . }
$$

Es lo que desde Heinze ${ }^{17}$ y Fraenkel ${ }^{18}$ vienen recononciendo los exégetas modernos ${ }^{19}$.

En lo que a métrica se refiere, 0 sol pulcer o laudande se corresponde, según digo, con la primera mitad de un septenario trocaico, de un versus quadratus: es cuantitativamente regular - el que los pies impares no sean puros (lo son los pares) resalta su carácter popular, en cuanto que la medida es por pies y no por metros dipódicos, como correspondería a una versificación culta -. El isosilabismo es en él absoluto, así como la regularidad acentual: ' $\sim \sim \sim^{\prime} \sim \sim^{\prime} \sim$ ' $\sim$. El octosílabo, además, se articula en los dos commata tetrasilábicos habituales en el quadratus ${ }^{20}$.

Y, si esto es así en lo métrico, en lo que a estilo se refiere las palabras de este colon se compadecen del todo con un canto popular que bajo su rústica sencillez encierra una profunda afectividad: sol puede entenderse en sentido propio o en metonimia por dies o incluso como una alusión simbólica a Augusto ${ }^{21}$. La repetición de $o$ en anáfora al comienzo de cada uno de los commata tiene un aire popular y recalca la normal estructura del quadratus. Llamativo asimismo es el vocativo laudande: los raros vocativos de gerundivo, restringidos a la lengua poética, no se documentan antes del período augústeo ${ }^{22}$.

5 Todo ello, además, ha sido sabiamente ajustado al ritmo silábico-acentual del sáfico. Y en este sentido cabe poner de manifiesto que, aun tratándose del libro cuarto, en el que, como es bien sabido, los sáficos articulados en sexta sílaba no son tan raros como en los tres primeros libros, se puede hablar de cierta «irregularidad» o «excepcionalidad» en la presencia que dicho corte tiene en el pasaje: la necesidad de acomodar al sáfico la exclamación triunfal o el fragmento del septenario que se cita habría hecho que nada menos que tres sáficos seguidos (el primero de ellos, tercero de su estrofa) presentaran dicho corte excepcional en sexta sílaba:

pulcer, o laudande' // canam recepto

Caesare felix.

teque, dum procedis, // io Triumphe,

non semel dicemus, // io Triumphe.

Nada, por tanto, impide pensar que Horacio recogió aquí un fragmento de un canto que realmente existió y se cantó.

Y no hay que olvidar, además, que la cita en cuestión se inserta nada menos que dentro del pindárico libro cuarto y precisamente en la oda segunda, donde se elogia la excelsitud de Píndaro.

Horacio, como empecé diciendo, desde su excelsa cumbre del romanae fidicen lyrae, que odia y mantiene lejos de sí al vulgo ignorante (odi profanum vulgus et arceo), daría aquí sobrada muestra de su conocimiento de la poesía popular. Bien entendido, no obstante, que el hecho de que el vate, siempre crítico frente al vulgus y aristocráticamente hostil a confundirse con él, termine por asociarse al vulgus en esta circunstancia no depende de un repentino ataque de modestia, sino que es una funcional oferta de homenaje aún más significativo al princeps: la alegría por el regreso de Augusto es de todos, no sólo de la plebe sino también del excelso poeta ${ }^{23}$. 
19 2.2. Pues bien, a mí me parece que este pasaje no es algo excepcional en los versos líricos de Horacio; creo que en ellos se pueden reconocer otros en los que el poeta ha recogido igualmente expresiones o incluso posibles citas de la lírica popular.

En algún caso estas expresiones aparecen como lírica colectiva (dicimus), en el contexto de una celebración religiosa: tal es el de carm. IV 5,37 ss.:

'longas o utinam, dux bone, ferias \|

praestes Hesperiae', dicimus ...

dicimus...

Aquí, no obstante, el posible eco de un versus quadratus popular sería muy lejano e inseguro: ¿cabría reconocer un colon trocaico en un supuesto longas praestes ferias?

Los cantos de los amantes desdeñados que pasaban las noches ante las puertas de la amada - cantos que, según Horacio, Lidia oía ya cada vez con menos frecuencia -, ¿se expresaban también a base del popular versus quadratus?:

... audis minus et minus iam || 'me tuo longas pereunte noctes || Lydia, dormis?':

carm. I 25,7 ss.

Podría el poeta haber entreverado en estos sáficos un verso más o menos como éste:

longas noctes, <heu> pereunte, + Lydia, dormis me tuo?

Ecos también lejanos de una posible secuencia cuadrimembre podrían adivinarse en carm. IV 11, en el entramado fónico y léxico-morfológico de estos cuatro commata de los versos (SAPH 11s) 16 ss.:

rídet argénto / dómus, ára cástis

víncta verbénis / ávet ìmmoláto $\|$

spárgier ágno

que se insertan en un contexto en modo alguno ajeno a un carmen ritual ${ }^{24}$ :

ára cástis $\mid$ víncta verbénis + ávet ìmmoláto $\mid$ spárgier ágno

Tales ecos, sin embargo, parecen otras veces más cercanos y seguros. Así diría yo que ocurre en los dos pasajes siguientes.

2.2.1. Uno es el del epodo noveno, al cual ya me he referido. En él los Galli canentes Caesarem y las mencionadas exclamaciones io Triunphe nos llevan de nuevo a los «cantos de triunfo»:

at huc frementis verterunt bis mille equos || Galli canentes Caesarem I|| hostiliumque navium portu latent || [20] puppes sinistrorsum citae. ||| io Triumphe, tu moraris aureos || currus et intactas boves? || io Triumphe, nec Iugurthino parem || bello reportasti ducem ||| neque Africanum, cui super Carthaginem || virtus sepulcrum condidit. ||| terra marique victus hostis punico || lugubre mutavit sagum, ||| aut ille centum nobilem Cretam urbibus || [30] ventis iturus non suis, $\|$ exercitatas aut petit Syrtis Noto \| aut fertur incerto mari $\|$ : epod. 9,17 ss.

Los gálatas abandonan a Antonio y se pasan a Octaviano - «César» en cuanto que hijo adoptivo del Gayo Julio César -, aclamándolo, o quizá mejor, «cantándole» (canentes). El pasaje $e^{25}$ parece ${ }^{26}$ dar cuenta de la costumbre de dichos soldados de cantar al entrar en combate:

omnium quae Asiam colunt gentium Gallos fama belli praestare. Inter mitissimum genus hominum ferox natio pervagata bello prope orbem terrarum sedem cepit. Procera corpora, promissae et rutilatae comae, vasta scuta, praelongi gladii; ad hoc cantus ineuntium proelium et ululatus et tripudia, et quatientium scuta in patrium quendam modum horrendus armorum crepitus, omnia de industria composita ad terrorem ${ }^{27}$. 
Aun así, dado el contexto y las frases que siguen, no es imposible pensar aquí en una especie de carmen triumphale. Al menos Horacio habría podido reinterpretar dicho canto desde la perspectiva de los carmina militaria o triumphalia latinos.

El grito ritual io Triumphe, con que los ciudadanos vitoreaban al general y sus soldados durante la procesión triunfa ${ }^{28}$, aparece como una interpelación a un dios personificado ${ }^{29}$. Dicho grito, al que, como acabamos de ver, recurriría luego Horacio en el libro cuarto de sus Odas, trasprantándolo al esquema del endecasílabo sáfico, ocupa aquí su lugar natural, el primer hemistiquio (penthemímeres yámbico) del trímetro:

$x--/-x-\sim \|$

io Triumphe, tu moraris aureos || currus et intactas boves? || io Triumphe, nec Iugurthino parem $\|$ bello reportasti ducem || $\mid:$ epod. 9,21 ss.

Y no hay duda de que semejante colon a cualquier oído de la época - como a cualquier conocedor moderno de la versificación latina- le trae ecos del final del primer miembro (dímetro o, mejor, cuaternario) de un septenario trocaico, el verso, como es bien sabido, de estos carmina triumphalia:

$-\quad \underline{x-}+-\quad-x-\quad \sim \|$.

Dichos septenarios se dejarían oír aquí claramente a través del trímetro yámbico, cuyos vínculos estructurales con el verso trocaico son indudables ${ }^{30}$.

En el primero de los dos versos en cuestión la acentuación es totalmente yambotrocaica:

'- '- i|ó Triúmphe, + tú moráris | áureòs;

en el segundo sólo lo es parcialmente (algo, por lo demás, que no se sale de la norma):

'- '- i|ó Triúmphe, + nec Iugurthi|no parem.

Es más, el dímetro yámbico que sigue como verso epódico a cada uno de estos trímetros es un octosílabo, similar al dímetro trocaico del primer colon de un septenario. Y casi se adapta a su esquema cuantitativo, salvo en la sílaba penúltima, en el yámbico breve y en el trocaico larga. Pero en lo que a acentos de palabra se refiere el dímetro yámbico de los versos 20, 22 y 24 es idéntico a un dímetro trocaico como el que hace de primer miembro de un versus quadratus; cosa que no ocurre en ninguno de los otros dímetros de la composición:

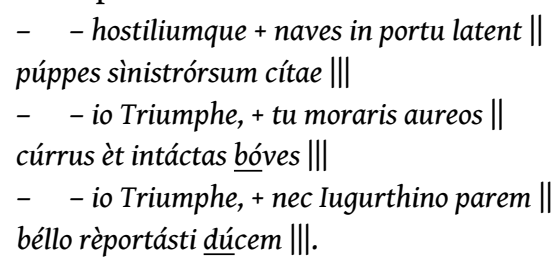

No resulta, por tanto, descabellado observar que, invirtiendo el orden de los hemistiquios y prescindiendo de ciertas irregularidades en el esquema cuantitativo, sería posible oír un septenario acentual:

cúrrus èt intáctas bóves + tú moráris áureòs?

Otro tanto en los otros dos, aunque sin regularidad acentual absoluta (cosa que también ocurre en los habitualmente reconocidos como tales):

púppes sìnistrórsum cítae + náves in portu latent

béllo rèportásti dúcem + nec Iugurthino parem.

2.2.2. El segundo de los pasajes a que me refería se halla en el epodo décimo séptimo, en el que se suele reconocer una cita irónica de un irónico verso de Catulo; así lo hacen tanto los comentaristas y estudiosos de Horacio ${ }^{31}$ como los del poeta de Verona ${ }^{32}$. 
31 Se trata de la expresión catulliana pudica et proba, que reaparece en Horacio como tu pudica, tu proba. Dicha expresión se halla en ambos en el contexto de una palinodia más o menos sarcástica. Catulo escribe en endecasílabos falecios:

Adeste, hendecasyllabi ... [10] circumsistite eam, et reflagitate: \| 'moecha putida, redde codicillos, $\|$ redde, putida moecha, codicillos!' $\|$... [18] conclamate iterum altiore voce: $\|$ 'moecha putida, redde codicillos, || redde, putida moecha, codicillos!' || sed nil proficimus, nihil movetur. || mutandast ratio modusque vobis, || siquid proficere amplius potestis, || ' pudica et proba, redde codicillos!': Catull. 42.

Horacio lo hace en trímetros yámbicos:

sive mendaci lyra || voles sonare / 'tu pudica, tu proba || perambulabis / astra sidus aureum' |||: Hor., epod. 17,39 ss.

Evidentemente el horaciano mendaci lyra resulta ambiguo (¿intencionadamente?) según se entienda referido al pasado (lira «que ha sido mendaz» cuando atacaba a Canidia) o al futuro («que va a ser mendaz» al alabarla); o, mejor dicho, le resultaría ambiguo a Canidia, que podría entenderlo como ella quisiera; para Horacio con toda probabilidad el sentido sería el segundo ${ }^{33}$. La alabanza, así, en él sería expresamente insincera; y más o menos lo mismo ocurría en Catulo.

Fordyce $^{34}$, que, como los demás admitía las reminiscencias catulianas del pasaje horaciano, advertía de la posibilidad de que tal expresión aliterante fuese la misma frase convencional («phrase ... probably conventional») que ya aparece en un verso de Afranio.

Y esta presencia de proba et pudica en Afranio abriría la posibilidad de reconocer lazos entre la palinodia de Horacio y la de Estesícoro, cosa que llevaría a referir el epodo horaciano no tanto a Catulo cuanto al poeta griego ${ }^{35}$.

Por otra parte, las raíces populares o folklóricas de la poesía de Catulo, algo reconocido por los estudiosos ${ }^{36}$, se hacen evidentes ${ }^{37}$ en este poema 42 , que en realidad se sustenta formalmente, según Goldberg ${ }^{38}$ sobre una flagitatio como la conocida del Pseudolus (357 ss.) plautino. El propio Goldberg ${ }^{39}$, seguido luego por Green $^{40}$, ha reconocido en el poema de Catulo reminiscencias de la comedia tanto en lo que respecta a la escansión de los versos (más específicamente, a la «composición») como en lo relativo al vocabulario.

El mencionado verso de Afranio es un septenario trocaico:

Nam proba et pudica quod sum, consulo et parco mihi: Afran. frg. 116 Ribbeck (vol. II, 1898).

$\mathrm{Y}$, en el camino que trato de seguir, trae a la memoria estos otros dos versos (respectivamente, septenario trocaico y senario yámbico) de Plauto y Terencio:

Plaut., Amph. 1086 (TRse)

Amphitruo, piam et pudicam esse tuam uxorem ut scias;

Ter., Adelph. 930 (IA6p)

[DE.] proba et modesta. [MI.] ita aiunt. [DE.] natu grandior.

Pues bien, así las cosas, ¿podría tratarse, entonces, de un fragmento de canción popular irónica (o utilizado irónicamente por Catulo)? La expresión pudica et proba tal como aparece en el verso catuliano ( - - ) no encaja muy bien en un rítmo yambotrocaico. Tampoco en Catulo hay alusión a canto alguno.

En Horacio, en cambio, sí se inserta el dicho en un contexto musical (sonare) que puede apuntar al canto. Y, además, tu pudica, tu proba puede ser muy bien un colon yambotrocaico, en concreto el segundo de un septenario. 
¿Podría pensarse, entonces, en un canto popular citado primero por Catulo (que en tal caso habría alterado el texto original para acomodarlo al esquema del falecio), y vuelto a citar por Horacio, pero en su forma auténtica yambo-trocaica?

Catulo, en efecto, escribe en falecios, un endecasílabo en el que, contra lo que ocurrió en los horacianos, sobre todo en el sáfico, no llegó a consolidarse en latín una articulación en dos miembros fijos. En Catulo, sin embargo, hay un predominio de cortes en quinta sílaba (55) seguidos de cerca por los cortes tras la sexta (41). En Marcial la relación se invertirá: $38 / 53^{41}$. Tanto uno como otro corte en el centro del verso suponen un segundo miembro de ritmo yambo-trocaico: bien similar a una tripodia trocaica («itifálico»: $\sim-\quad / \quad-\quad-\sim)^{42}$, bien equivalente a un colon yámbico penthemímeres, como el habitual en el trímetro $(\sim \sim-\quad-/ \quad-\quad-\sim)^{43}$.

2 En el poema 42 llama la atención la extraordinaria frecuencia del corte en quinta sílaba, que aparece nada menos que en veinte ${ }^{44}$ de sus veinticuatro versos, dejando así sentir con fuerza la presencia de un segundo colon trocaico ${ }^{45}$ : es además la articulación que presenta el verso que nos ocupa (24) pudic(a et proba / redde codicillos, que, a su vez, se hace eco de los anteriores, 19 y 20 moecha putida / redde codicillos, $\|$ redde, putida / moecha, codicillos.

Las aliteraciones y repeticiones son, como en los versos de la comedia, propias de la poesía popular ${ }^{46}$.

4 En Horacio, como acabo de decir, la cosa sería aún más sencilla y directa: las palabras del originario canto popular irían tal cual, trasplantadas desde el segundo miembro (cuaternario trocaico cataléctico) de un septenario, al segundo de un trímetro yámbico con su normal cesura penthemímeres, hermano gemelo suyo, como he dicho.

En conclusión, detrás del horaciano:

voles sonare / 'tu pudica, tu proba || perambulabis / astra sidus aureum' podría estar un canto popular anónimo:

- - - - + tu pudica, tu proba $\|$

- - perambulabis + astra sidus aureum $\|$.

La repetición anafórica del tu encabezando los dos metros del hemistiquio, sería una vez más la propia de los cantos populares ${ }^{47}$. La absoluta homodinia apuntaría en el mismo sentido ${ }^{48}$.

\section{BIBLIOGRAFÍA}

AUGELLO G. 1991, «Catullo e il folklore. La flagitatio nel c. 42», in Studi di filologia classica in onore di Giusto Monaco, Palermo, pp. 723-735.

CAPELLE W. 1929, «Zu Tacitus’ Archäologien», Philologus 84, pp. 201-208.

CORTÉS R., FERNÁNDEZ CORTE J.C. (eds) 1994, Bimilenario de Horacio, Acta Salmanticensia. Estudios filológicos 258, Salamanca.

COURTNEY E. (ed.), The Fragmentary Latin Poets, Oxford, 1993. 
DEL GRANDE C. 1960, Enciclopedia classica. Sezione II, Lingua e letteratura. Vol. V, La Lingua greca nei mezzi della sua espressione. T. II, La metrica greca, Torino.

Della CoRTe F. (ed.) 1977, Catullo. Le Poesie, Scrittori greci e latini, Milano.

Dolç M. (ed.) 1963, Catulo. Poesías, Col. hispánica de autores griegos y latinos, Madrid.

ELLIS R. 1889, A Commentary on Catullus, 2da ed., Oxford.

FEDELI P. (ed.) 1994, Q. Orazio Flacco. Le opere. II, Le satire, Antiquitas perennis, Roma, 2 vol.

FEDELI P. (ed.) 1997, Q. Orazio Flacco. Le opere. II, Le epistole; L'arte poetica, Antiquitas perennis, Roma. FEDELI P., CICCARELlI I. (eds) 2008, Q. Horatii Flacci Carmina. Liber IV, Serie dei classici greci e latini. Testi con commento filologico NS 17, Firenze.

FERGUSON J. 1956, «Catullus and Horace», AJPh 77, pp. 1-18.

FERNÁNDEZ CORTE J.C. 1994, «Catulo en Horacio», in R. Cortés, J.C. Fernández Corte (eds), Bimilenario de Horacio, Acta Salmanticensia. Estudios filológicos 258, Salamanca., pp. 39-61.

FERNÁNDEZ CORTE J.C., GONZÁLEZ IGLESIAS J.A. (eds) 2006, Catulo. Poesías, Letras universales 304, Madrid. FORDYCE C.J. 1961, Catullus: A Commentary, Oxford.

FRAENKEL E. 1927, «Die Vorgeschichte des Versus quadratus», Hermes 62, pp. 357-370 (= Fraenkel 1964, II, pp. 11-24).

FRAENKEL E. 1957, Horace, Oxford.

FRAENKEL E. 1961, «Two Poems of Catullus», JRS 51, pp. 46-53 (= Fraenkel 1964, II, pp. 115-129).

FRAENKEL E. 1964, Kleine Beiträge zur klassischen Philologie, Storia e letteratura 95-96, Roma, 2 vol.

GERICK T. 1996, Der versus „quadratus” bei Plautus und seine volkstümliche Tradition, ScriptOralia. Reihe A, Altertumswissenschaftliche Reihe 21, Tübingen.

GOLDBERG S. 2000, «Catullus 42 and the comic legacy», in E. Stärk, G. Vogt-Spira (eds) 2000, Dramatische Wäldchen: Festschrift für Eckard Lefevre zum 65. Geburtstag, Spudasmata 80, Hildesheim, pp. 475-489.

GRANAROLO J. 1967, L'œuvre de Catulle : aspects religieux, éthiques et stylistiques, Coll. d'études anciennes, Paris.

GREEN P. (ed.) 2005, The Poems of Catullus: A Bilingual Edition, Berkeley.

HARRIS W. 2011, «Catullus Poem 42», in Humanities and the Liberal Arts <http://

community.middlebury.edu/ harris/catullus.42.html>.

KASSEL R. 1981, «Dichterspiele», ZPE 42, pp. 11-20.

KIESSLING A. (ed.) 1917, Q. Horatius Flaccus, 6. Aufl. erneuert von R. Heinze, Sammlung griechischer und lateinischer Schriftsteller mit deutschen Anmerkungen, Berlin, 3 vol.

LAFAYE G. (ed.) 1923, Catulle. Poésies, CUF, Paris.

LINDO L.I. 1969, «Horace's seventeenth Epode», CPh 64, 3, pp. 176-177.

LoomIS J.W. 1972, Studies in Catullan Verse: An Analysis of Word Types and Patterns in the Polymetra, Mnemosyne: bibliotheca classica Batava. Suppl. 24, Leiden.

LUQUE MORENO J. 2009, Versus quadratus: crónica milenaria de un verso popular, Biblioteca de humanidades. Estudios clásicos 26, Granada. 
LUQUE MORENO J., LÓPEZ EISMAN A. (eds) 2007, San Agustín. Sobre la música: seis libros, Biblioteca clásica 359, Madrid.

MCNEILL R.L.B. 2007, «Catullus and Horace», in M.B. Skinner (ed.), A Companion to Catullus, Blackwell Companions to the Ancient World, Malden, pp. 357-376.

NIEBUHR B.G. 1843, Kleine historischen und philologischen Schriften, II, Berlin.

PEDICONE J. 2010, «Moecha Metrica: Meter Games in Catullus 42», APAClassics, American Philological Association.

PÉREZ VEGA A. 2003, «El 'Libro del passer’ de Catulo: notas de lectura», Exemplaria 7, pp. 79-94.

PÉREZ VeGA A., RAMírez De Verger A. (eds) 2005, C. Valerii Catulli Carmina = Catulo. Poemas, Huelva.

QUINN K. (ed.) 1970, Catullus: The Poems, London (1973²).

Romano E. 1991, Q. Orazio Flacco. Le opere. I, Le odi; Il carme secolare; Gli epodi, Roma, 2 vol.

RONCONI A. 1971, Studi catulliani, nuova ed., Antichità classica e cristiana 8, Brescia.

ROSTAGNI A. 1964, Storia della letteratura latina, 3. ed., riv. e ampl. a cura di I. Lana, Torino, 3 vol.

SKINNER M.B. (ed.) 2007, A Companion to Catullus, Blackwell Companions to the Ancient World,

Malden.

SOLER RUIz A. (ed.) 1993, Catulo. Poemas; Tibulo. Elegías, Biblioteca clásica 188, Madrid.

SOUBIRAN J. 1988, Essai sur la versification dramatique des romains : sénaire iambique et septénaire trochaïque, Paris.

STÄRK E., VOGT-SPIRA G. (eds) 2000, Dramatische Wäldchen: Festschrift für Eckard Lefevvre zum

65. Geburtstag, Spudasmata 80, Hildesheim.

STEPHENSON L. 1997, «Urbanitas in Some Poems of Catullus $(1,13,10,86,42,12,39,84,36,22) »$, Classicum 23, 2, pp. 41-50.

STRECKER H. 1950, Die volkstümliche Elemente in den kleineren Gedichten des Catull [Diss. Jena].

THOMAS R.F. (ed.) 2011, Horace. Odes Book IV and Carmen Saeculare, Cambridge Greek and Latin Classics, Cambridge.

USENER H. 1901, «Italische Volksjustiz», RhM 56, pp. 1-27 (= Kleine Schriften, IV, pp. 356 ss.)

VERSNEL H.S. 1970, Triumphus: An Inquiry into the Origin, Development and Meaning of the Roman

Triumph, Leiden.

VIPARELLI V. 1998, s.v. «Monsillabi finali», in Orazio: enciclopedia oraziana. II, La culura, la società, la poesia.

WATSON L.C. 2003, A Commentary on Horace's Epodes, Oxford.

\section{NOTAS}

1. Siempre con mal gusto: carm. II 13,31 ... sed magis $\|$ pugnas et exactos tyrannos $\|$ densum umeris bibit aure volgus.

2. Sat. I 5,50 ss. hinc nos Coccei recipit plenissima villa, || quae super est Caudi cauponas. nunc mihi paucis || Sarmenti scurrae pugnam Messique Cicirri, || Musa, velim memores et quo patre natus uterque \| contulerit litis. Messi clarum genus Osci; || Sarmenti domina exstat: ab his maioribus orti || ad pugnam 
venere. prior Sarmentus 'equi te $\|$ esse feri similem dico.' ridemus, et ipse \| Messius 'accipio,' caput et movet. 'o tua cornu \| ni foret exsecto frons,' inquit, 'quid faceres, cum $\|$ sic mutilus minitaris?

3. Etrusco de nacimiento (natione Tuscus), según los escolios a Juvenal $(5,3)$, habría sido esclavo del pompeyano Favonio y después de Mecenas, el cual lo habría liberado; Juvenal lo recuerda como conocidísimo parásito. Es con éste - y no con el favorito de Antonio, al que se refiere Plutarco, Ant. 59 - con el que se suele identificar el Sarmentus de Horacio: cf., por ejemplo, Fedeli 1994, p. 429.

4. FPL, pp. 248 ss; Courtney 1993, pp. 475 s.

5. Porph., ad epist. I 1,62, p. 319,4 Holder contra pueri lusu cantare solent rex ... non erit Quam neniam meliorem ait, quia non divitiis, se virtutibus honor habeatur.

Cf. asimismo Ps.Acron, ad loc., p. 211 Keller Idest etiam pueri dicunt summum bonum esse virtutem. Aliter: etiam pueri dicunt: qui recte vivit rex est; Isid., orig. X 3,4; Fraenkel 1964, II, 18; Kassel 1981; Fedeli 1997, p. 1001, según el cual, la eficacia del 'ritornello', que tiene antecedentes en juegos análogos de los niños griegos, entre los que el líder era llamado 'rey', se basa en el juego etimológico entre rex y recte.

6. FPL 10, p. 417; Courtney 1003, pp. 484 s.; Luque 2009, p. 29.

Más que la opción por la segunda o la tercera persona, es digna de atención la sabia adaptación de la fórmula desde el ritmo trocaico $(-|--|--\mid \quad-$, primer miembro de un septenario popular -no dímetro culto) al dáctilico $(-\quad|-|--|--|-|--||--|-\quad \mid-\ldots$

7. Porph., ad ars 417, p. 177, 11 Holder hoc ex lusu puerorum sustulit, qui ludentes solent dicere: quisquis ad me novissimus venerit, habeat scabiem (Müller: habeat scabiem quisquis ad me venerit novissimus).

Ps.Acrón, ad loc., p. 373,6 Keller $\mu \varepsilon \tau \alpha \varphi \circ \rho\llcorner\kappa \tilde{\omega} \varsigma$ locutus est a ludo puerorum. Ita enim pueri currentes: 'occupet scabies in extremo remanentem'. Id est ego poeta sum, habeat scabiem qui non est poeta.

8. FPL 11, p. 417; Luque 2009, p. 29.

9. Sobre la práctica por parte de los versificadores de estos juegos de transposición rítmica, cf. Kassel 1981.

10. Cf. epist. II 1,157 .

11. Suet., Aug. 57.

12. Ps.Acron, ad loc., p. 333 Keller: ad ipsum quasi triumphum loquitur sacra invocatione, qua 'io, io' dicebatur, ut (Verg., Aen. VII 400) Io matres, audite; Porph., ad loc., p. 141,18 Holder: Ad ipsum triumphum conversus haec dicit.

13. Recuérdense las palabras de Suetonio (Cal. 6.): undique concinentium 'salva Roma, salva patria, salvus est Germanicus'.

14. Cf., por ejemplo, Romano 1991, p. 859.

15. Niebuhr 1843, p. 259.

16. El monosílabo en final de verso contribuiría a marcar la imagen del ritmo trocaico subyacente: cf. Viparelli 1998, p. 921.

17. Kiessling 1917, p. 409, ad loc.

18. Fraenkel 1927, p. 364, n. 1.

19. Por ejemplo, Fraenkel 1957, pp. 438 s.; Kassel 1981, pp. 17 s.; Romano 1991, p. 859; Fedeli, Ciccarelli 2008, pp. 165 s.; Thomas 2011, p. 118.

20. Cf. Luque 2009.

21. Fedeli, Ciccarelli 2008, pp. 165 s.

22. Thomas 2011, p. 118. Fedeli sugería la posibilidad de que este laudandus se anunciara ya antes de forma anagramática en el verso nueve: laurea donandus Apollinari.

23. El mismo concepto aparece luego en carm. IV 5,9 ss. Fedeli, Ciccarelli 2008, p. 170.

24. Cf. Thomas 2011, ad loc.

25. Al que se le puede encontrar parelelo en Verg., Aen. VII 698 ibant aequati numero regemque canebant o en Sil. VIII 420 ibant et laeti ... Sancum voce canebant. 
26. Cf. Watson 2003, ad loc.

27. Liv. XXXVIII 17, 4 s., referido a estos galos de Asia en 189 a.C. Cf. asimismo Tac., Germ. 3 Sunt illis haec quoque carmina, quorum relatu, quem barditum vocant, accendunt animos futuraeque pugnae fortunam ipso cantu augurantur. Terrent enim trepidantve, prout sonuit acies, nec tam vocis ille quam virtutis concentus videtur (Sobre este pasaje tacíteo, cf. Capelle 1929).

28. Varr. LL VI 68; Hor., carm. IV 2, 49 s.; Ov., am. I 2,34; Mart. VII 6,7. Cf. Versnel 1970, pp. 30-48.

29. Porph., ad vv. 21 s., p. 203,13 Holder Quasi deum invocat triumphum. Tu, inquit, moraris currus, qui iam parati sunt; tandem veni. Recuérdense asimismo los menionados pasajes del Pseudo Acrón y del propio Porfirión a propósito del correspondiente pasaje de la oda segunda del libro cuarto.

30. El trímetro sin su crético inicial es igual que el septenario; sus correspondencias en el nivel de los esquemas son claras; tal como, ya en el plano de la composición, lo son otras peculiaridades de tipología verbal o, sobre todo, la equivalencia entre la juntura central del septenario y la cesura penthemímeres del trímetro: cf., por ejemplo, Soubiran 1988.

31. Por ejemplo, Romano 1991, ad loc.; Watson 2003, ad loc.; cf asimismo Lindo 1969, p. 176.

32. Cf., por ejemplo, las observaciones ad locum de Ellis 1889; Fordyce 1961 o Quinn 1970; cf. asimismo Fraenkel 1961; Augello 1991; Pérez Vega 2003, pp. 88 s.; McNeill 2007, p. 368.

33. Cf. Plessis, Lejay, ad loc.

34. Fordyce 1961, ad loc.

35. Cf. Lindo 1969, quien, a mi juicio, aducía razones más que suficientes para ello.

36. Recuérdense, por ejemplo, Strecker 1950; Rostagni 1964, p. 471; Granarolo 1967, p.166; Ronconi 1971, pp. 65 ss.

37. Cf., por ejemplo, Usener 1901; Fraenkel 1961; Augello 1991.

38. Goldberg 2000.

39. Ibid., pp. 481 ss.

40. Green 2005, p. 226

41. Cf. Loomis 1972, p. 44.

42. Era la interpretación del falecio dominante en Aftonio (Mar. Vict., GLK VI 118,10), quien recoge incluso como denominación alternativa habitual de este verso la de «itifálico»: Phalaecium ... quod et ithyphallicum fit, si tribus trochaeis claudatur ... idem fiet ithyphallicum sub ea divisione in qua primus pes ... erit ... quidam tamen nullam volunt esse differentiam inter hos, sed eundem ithyphallicum et phalaecium accipiunt.

Algo parecido se lee en Terenciano Mauro (vv. 2545 ss.) Quem nos hendecasyllabum solemus $\|$... vocare ... || spondeon siquidem videmus istic || tamquam legitimus solere poni $\|$, post hunc dactylon atque tres trochaeos, || cui nomen quoque phallico dederunt.

Es la «segunda división» del falecio que se recoge en la larga exposición sobre el verso que figura en Basso/Fortunaciano: Caes. Bass. [Atil. Fort.], GLK VI 258,25: Altera divisio est ... hoc exemplo siccas ducite machinae carinas. nam primi duo pedes ... reliqua pars ithyphallicum facit.

Sobre estas interpretaciones o «divisiones» del falecio (todas ellas desde la falsa perspectiva de la versificación jónica por pies y de la métrica alejandrina), difundidas entre los artígrafos tardíos y mantenidas luego por muchos estudiosos modernos, cf., por ejemplo, Del Grande 1960, pp. 328 s.

43. Que se uniría a un primer miembro pentemímeres dactílico: cf., por ejemplo, Terent. Maur. 2674 ss. (hendecasyllabus) ... componitur ex utroque metro. || pars heroica tum prior ... || ut sit pentameris tome locata; || exin cetera portio est iambi; Caes. Bass. [Atil. Fort.], GLK VI 258, 27 ss. hendecasyllabum phalaecium ... prima vulgaris illa divisio, quae docet eum partem habere ex heroo, parte ex iambo, cuius exemplum castae Pierides meae Camenae

44. Contando también el verso primero Adest(e hendeca/syllabi quot estis.

45. Algo en lo que Goldberg (2000, pp. 480 s.) reconocía uno de los vínculos del poema de Catulo con la comedia anterior.

46. Merecería quizá también nuestra atención la advertencia que hace el poeta a sus hendecasyllabi, interlocutores suyos en todo el poema, en el verso 22: mutandast ratio modusque 
vobis; y en ella, sobre todo, la expresión ratio modusque, que parece una frase hecha ( ${ }^{\mathrm{f}} \mathrm{f}$., por ejemplo, Hor., serm. II 3, 266 y 271; epist. II 1, 20) y en la que al término modus no se le reconoce con frecuencia su sentido musical (Ramírez 1988: «habrá que cambiar de modos y maneras»; Soler 1993: «Tenéis que cambiar de idea y de método»; Pérez Vega, Ramírez 2005: «debéis cambiar la forma y la manera»; Green 2005: "you'll need to change your tune and method»; González Iglesias 2006: «hay que cambiar el plan y los modales»). Yo, entendiéndola en la línea de Lafaye (1923: «Il nous faut changer de système et de ton») y Dolç (1963: «Tenemos - nobis - que cambiar de sistema y de tono») o, sobre todo, de Della Corte (1977: «Bisognera che cambiate tono e musica»), preferiría traducirla «tendréis que cambiar de plan y de aire».

Creo, en efecto, que hay que distinguir entre ratio (método, plan general, sistema de ataque) y modus (forma musical) y reconocerle a este último su sentido métrico-musical, es decir, su significado de «medida», «patrón» o estructura rítmico-melódica (Cf., por ejemplo, Luque, López 2007, pp. 90 s., n. 9 s.). Más o menos lo que nosostros designamos como « aire »: cf. Catull. 61,38 vosque item simul, integrae || virgines, quibus advenit || par dies, agite in modum \| dicite, 'o Hymenaee Hymen; 126 ite concinite in modum || 'io Hymen Hymenaee io;

Hor., epist. I 19,26 ac ne me foliis ideo brevioribus ornes $\|$ quod timui mutare modos et carminis artem; carm. II 1,40; 9,9; 12,4; III 3,72; 9,10; 11,8; 30,14; IV 6,43; 11,34 .

De esa manera se podría reconocer con Pedicone (2010) en este verso 22 un anuncio y una preparación de lo que va a ocurrir en el verso 24 , donde, tras los endecasílabos de base trocaica moecha putida (11 y 19) y redde putida (12 y 20) se va a cambiar a uno de base yámbica: pudica et proba; uno distinto, por tanto, no sólo en el contenido (de acuerdo con la palinodia, laudatorio frente a los anteriores insultos), sino también en lo métrico: como si el poeta hubiese querido dejar constancia de que introduce algo nuevo, ajeno en cierto modo a los anteriores falecios.

47. Watson (2003, ad loc.) entiende la anáfora de segunda persona como característica de los himnos.

48. ¿Cabría oír un septenario silábico-acentual en una supuesta secuencia: tú proba peràmbulábis + ástra sídus áureùm?

\section{RESÚMENES}

Bien qu'Horace semble récuser tout auditoire populaire pour sa poésie lyrique, il est possible d'y déterminer des emprunts plus ou moins déguisés à la poésie populaire. À travers plusieurs exemples, l'article montre l'utilisation de formules issues de la lyrique populaire et leur adaptation qui les intègre dans une poésie savante tout en laissant voir leur origine autre. 
ÍNDICE

nomsmotscles Afranius, Aphthonius, Caesius Bassus, Catulle, Horace, Juvénal, Martial, Ovide, Plaute, Plutarque, Porphyre, Pseudo-Acron, Silius Italicus, Stésichore, Suétone, Térence, Terentianus Maurus, Tite-Live, Varron, Virgile

Keywords: Latin comedy, ancient commentary, metric awareness, Latin grammarians, Greek and Latin metric, prosody, Latin accentuation, saturnian verse, carmen triumphale, choral songs, folklore, popular Roman lyric, accentual versification

Mots-clés: accentuation latine, carmen triomphale, chant choral, comédie latine, commentaire antique, conscience métrique, folklore, grammairiens latins, lyrique populaire romaine, métrique gréco-latine, prosodie, saturnien (vers), versification accentuelle

\section{AUTORES}

\section{JESÚS LUQUE MORENO}

Univ. de Granada 